\title{
Enhancing the Accuracy of Computerized Interpretation of Old Inferior Wall Myocardial Infarction in a Routine or Preoperative Electrocardiogram by Analyzing Repolarization Abnormalities and Q Waves in Leads II, III and aVF
}

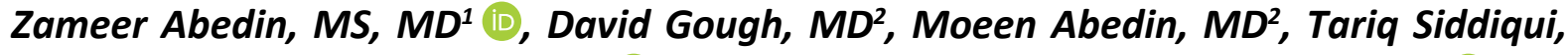 \\ $M D^{2}$, Debabrata Mukherjee, $M D^{2}$ (D), Chandra P Ojha, MD², Zuber D Mulla, PhD ${ }^{3}$ (D) and \\ Zainul Abedin MD, FRCP(C), FHRS ${ }^{*}$ (i) \\ ${ }^{1}$ Department of Internal Medicine, University of Utah School of Medicine, USA \\ ${ }^{2}$ Department of Internal Medicine, Paul L Foster School of Medicine, Texas Tech University Health Sciences Center, USA \\ ${ }^{3}$ Office of Faculty Development, Paul L Foster School of Medicine, Texas Tech University Health Sciences Center, USA
}

*Corresponding author: Zainul Abedin, MD, FRCP(C), FHRS, Professor of Clinical Medicine, Department of Internal Medicine, Paul L Foster School of Medicine, Texas Tech University Health Sciences Center, 4800 Alberta Ave, El Paso, TX 79905, USA

\section{Abstract}

Introduction: A diagnosis of an old inferior wall myocardial infarction (IMI) by computerized interpretation of a routinely performed electrocardiogram (ECG) may lead to further consultations and imaging studies to confirm or refute that diagnosis.

This study was designed to assess the value of abnormal $Q$ waves combined with repolarization abnormalities in inferior leads for the diagnosis of an old IMI as confirmed by imaging studies.

Methods: Fifty-six patients, in whom computerized interpretation of the ECG resulted in the diagnosis of old IMI, and in whom imaging studies were also available, were included in this study. ECGs were interpreted using the MUSEGE system. These ECGs were also analyzed by manually measuring the depth and the width of the $Q$ waves and morphology of ST and T-wave changes in inferior leads. Echocardiograms and nuclear medicine cardiac imaging were performed and interpreted using standard equipment and techniques. Measures of accuracy were calculated and reported with $95 \%$ exact binomial confidence intervals $(\mathrm{Cl})$.

Results: Computerized interpretation of the ECGs leading to the diagnosis of old IMI when compared with IMI confirmed by imaging studies, had a positive predictive value of
\end{abstract}

$52.8 \%$ (95\% exact $\mathrm{Cl}: 35.5 \%-69.6 \%)$. Adding the manually measured $Q$ waves wider than $40 \mathrm{msec}$ and amplitude of $-0.2 \mathrm{mV}$ and repolarization abnormalities in leads II, III and aVF increased the positive predictive value of the test to $86.2 \%$ (95\% exact $\mathrm{Cl}$ : $68.3 \%-96.1 \%$ ).

Conclusions: These results suggest that the computerized interpretation of ECG results in a high rate of false positive readings of old IMI. Presence of diagnostic $Q$ waves in inferior leads, if accompanied by repolarization abnormalities, improves the accuracy of the ECG for the diagnosis of old IMI.

\section{Keywords}

Inferior myocardial infarction, $Q$ waves myocardial infarction, Repolarization abnormalities, $Q$ waves: positive predictive value, Preoperative electrocardiogram

\section{Introduction}

Often, in the routinely or preoperative 12-lead electrocardiogram (ECG), the presence of $Q$ waves in inferior leads (Leads II,III, aVF), results in a computerized interpretation of an old inferior wall myocardial infarction (IMI). This leads to further consultations, imaging stud-

Citation: Abedin Z, Gough D, Abedin M, Siddiqui T, Mukherjee D, et al. (2020) Enhancing the Accuracy of Computerized Interpretation of Old Inferior Wall Myocardial Infarction in a Routine or Preoperative Electrocardiogram by Analyzing Repolarization Abnormalities and Q Waves in Leads II, III and aVF. Int J Clin Cardiol 7:196. doi.org/10.23937/2378-2951/1410196

Accepted: August 12, 2020; Published: August 14, 2020

Copyright: (C) 2020 Abedin Z, et al. This is an open-access article distributed under the terms of the Creative Commons Attribution License, which permits unrestricted use, distribution, and reproduction in any medium, provided the original author and source are credited. 


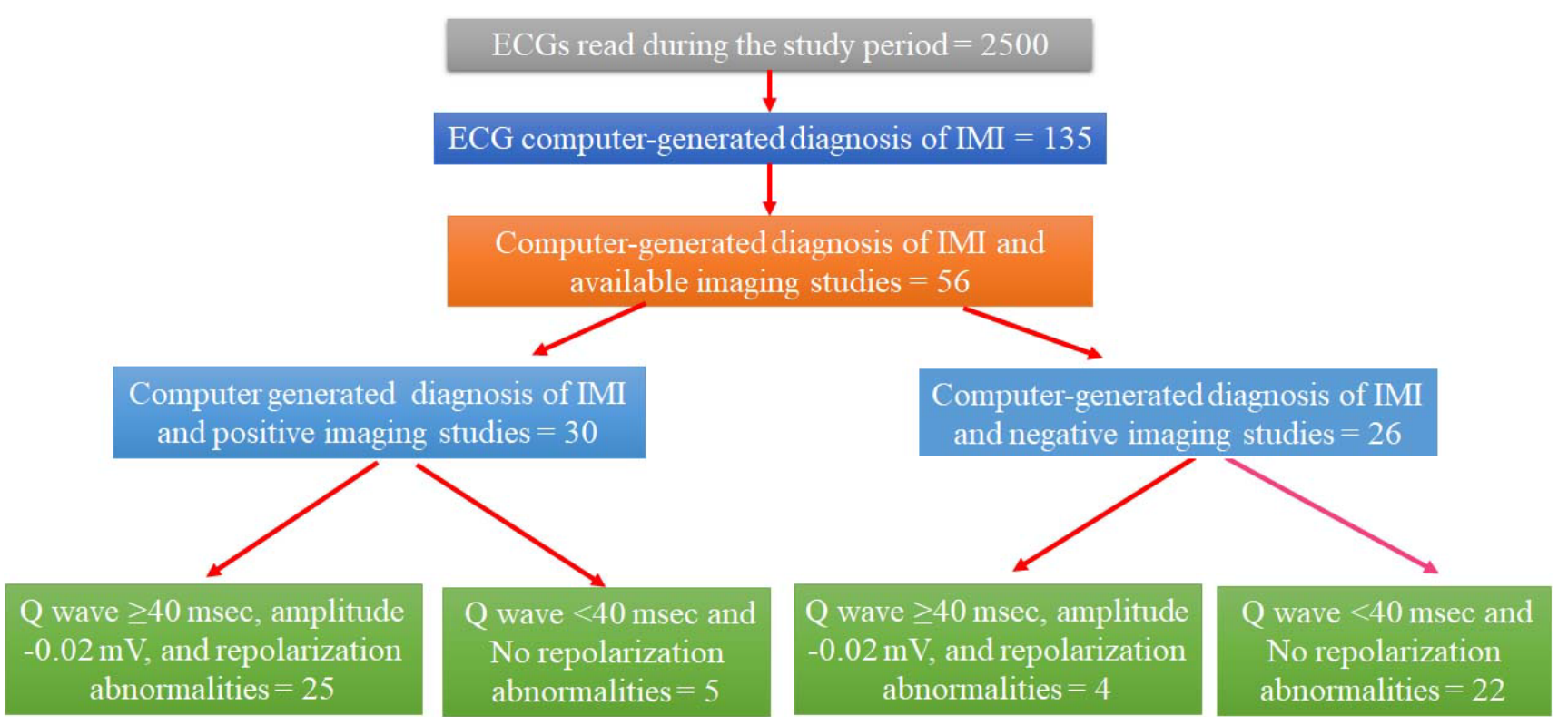

Figure 1: Flowchart, findings of the abnormal ECG and imaging studies.

ies, additional costs, rescheduling of the procedures and psychological stress for patients.

The diagnosis of a prior IMI is made if there are pathological $Q$ waves in the inferior leads of the ECG with or without symptoms of myocardial ischemia and imaging studies suggest a loss of viable myocardium in the same region [1-3]. A history of a prior myocardial infarction (MI) diagnosis, confirmed by elevated biomarkers is also an indicator of a previous MI.

Typically the width (duration) and depth (amplitude) of the $Q$ wave is used for the diagnosis of new or previous MI.Q waves are considered pathological when wider than 40 msec in duration and have an amplitude greater than $-0.2 \mathrm{mV}$ [4]. Despite general reliance on these ECG findings, there have been no recent studies to assess the predictive value of the $Q$ waves and repolarization abnormalities (ST and T wave) in inferior leads for the diagnosis of old IMI in a routine ECG performed in ambulatory settings.

The aim of this study was to assess the predictive value of abnormal Q-waves, and the role of repolarization abnormalities manifested by ST changes and negative or isoelectric T waves in inferior leads in improving accuracy of the ECG for the diagnosis of old IMI. To achieve this aim, ECG findings were correlated with myocardial scintigraphy, echocardiograms and/or coronary and left ventricular angiograms.

\section{Materials and Methods}

Two thousand five hundred, routinely performed, electrocardiograms were read during the study period of 2 years. Out of these 135 electrocardiograms with a computer-generated diagnosis of old IMI were identified (Figure 1). An IRB approval was granted (IRB \# E15047, submission reference \# 059266) using an expedited review process. Consent requirement was waived as this was a retrospective study.

Inclusion criteria were a computer-generated diagnosis of an old IMI on an electively performed 12-lead ECG and availability of an echocardiogram, coronary angiograms or nuclear perfusion scan performed in the last one year. Routine ECGs were performed in ambulatory settings. Some of these ECGs were performed for preoperative evaluation. Patients with acute or evolving myocardial infarction were not included. Electrocardiograms were recorded using normal standardization (10 $\mathrm{mm} / \mathrm{mV}), 25 \mathrm{~mm} / \mathrm{sec}$ speed by the GE MAC - HD 5500 machine. Computerized interpretations of the ECGs were performed using the MUSE GE system. If a patient had multiple tracings, the most recent one was included in the analysis. Manual measurements of the width and the depth of $Q$ waves in inferior leads (leads II, III, or aVF) were performed. A $Q$ wave duration of $\geq 40 \mathrm{msec}$ and a depth of the $Q$ wave of $\geq-0.2 \mathrm{mV}$ were considered suggestive of an old IMI. In addition, the deviation of the ST segment from the baseline by more than $1 \mathrm{~mm}$ and polarity of the $T$ wave were also analyzed. Abnormal $Q$ waves and repolarization abnormalities as defined above had to be present in at least two of the three inferior leads.

Echocardiograms, cardiac nuclear medicine imaging and cardiac catheterization coronary angiography were interpreted using standard equipment and techniques. The most recently performed imaging studies were included. Echocardiograms were analyzed for the presence or absence of inferior wall motion abnormalities and wall thickness. Cardiac scintigraphy images were analyzed for inferior wall defects. Defects due to diaphragmatic attenuation were excluded. Coronary angiograms were analyzed for occlusion of the coronary artery accompanied by angiographic evidence of left ventricular inferior wall motion abnormalities such as 
hypokinesis or akinesis.

Fifty-six patients, who had computer-generated ECG diagnosis of old IMI and who also had imaging studies performed within one year from the index electrocardiogram, were included.

There was no conflict of interest in acquisition of data, conduction of research and final preparation and submission of this manuscript.

\section{Data Analysis}

Demographic data of the sample were summarized. The accuracy of the computerized interpretation of the ECG was assessed by using imaging studies (echocar-

Table 1: Distribution of the patients with computer-generated diagnosis of inferior wall myocardial infarction and imaging studies.

\section{Echocardiogram, Nuclear Med Study, Coronary} LV Angiogram

ECG

\begin{tabular}{|l|l|l|l|} 
& Positive & Negative & Total \\
\hline Positive & 19 & 17 & 36 \\
\hline Negative & 11 & 9 & 20 \\
\hline Total & 30 & 26 & 56 \\
\hline
\end{tabular}

Table 2: Accuracy of computerized ECG for the diagnosis of an old inferior wall myocardial infarction vs. imaging studies.

\begin{tabular}{|l|l|l|}
\hline Measures & $\begin{array}{l}\text { Value } \\
\text { (\%) }\end{array}$ & $\begin{array}{l}\text { 95\% Exact } \\
\text { Confidence Interval }\end{array}$ \\
\hline Sensitivity & 63.3 & $43.9-80.1$ \\
\hline Specificity & 34.6 & $17.2-55.7$ \\
\hline Positive Predictive Value & 52.8 & $35.5-69.6$ \\
\hline Negative Predictive Value & 45.0 & $23.1-68.5$ \\
\hline
\end{tabular}

diogram or nuclear medicine imaging or coronary and left ventricular angiography) as the gold standard. The following measures of accuracy were calculated: sensitivity, specificity, positive predictive value (PPV), and negative predictive value (NPV). The PPV was also calculated for the computerized interpretation of the ECG combined with the presence of $Q$ waves wider than 40 msec with amplitude of $-0.2 \mathrm{mV}$ and repolarization abnormalities. SAS 9.4 software (SAS Institute, Inc., Cary, North Carolina) was used to calculate $95 \%$ exact binomial confidence intervals $(\mathrm{Cl})$ for the measures of accuracy listed above.

\section{Results}

Electrocardiograms with computer-generated diagnosis of old IMI were identified in 135 patients (Figure 1). Diagnostic imaging studies were available in 56 of 135 patients, 30 of these patients had abnormal studies (Table 1). Among the 56 patients included in this study, the average age was 63 years, and 38 (67.8\%) were male. The PPV was $52.8 \%(19 / 36)$ and the $95 \%$ exact $\mathrm{Cl}$ was 35.5-69.6. Sensitivity, specificity, and negative predictive value were calculated (Table 2).

In patients, whose ECG carried a computer-generated diagnosis of old IMI, manual measurement of the width and depth of the $\mathrm{Q}$ wave and ST segment and T wave analysis was performed. In 29 patients abnormal $Q$ waves and repolarization abnormalities, characterized by ST segment changes or T-wave inversions in at least two of the three inferior leads were noted (Figure 2). Twenty-five patients who had abnormal $Q$ waves and repolarization abnormalities also had positive imaging studies resulting in a PPV of $86.2 \%$ (95\% exact $\mathrm{Cl}$ : $68.3 \%$ $96.1 \%)$. Four patients (16\%) had abnormal Q waves and

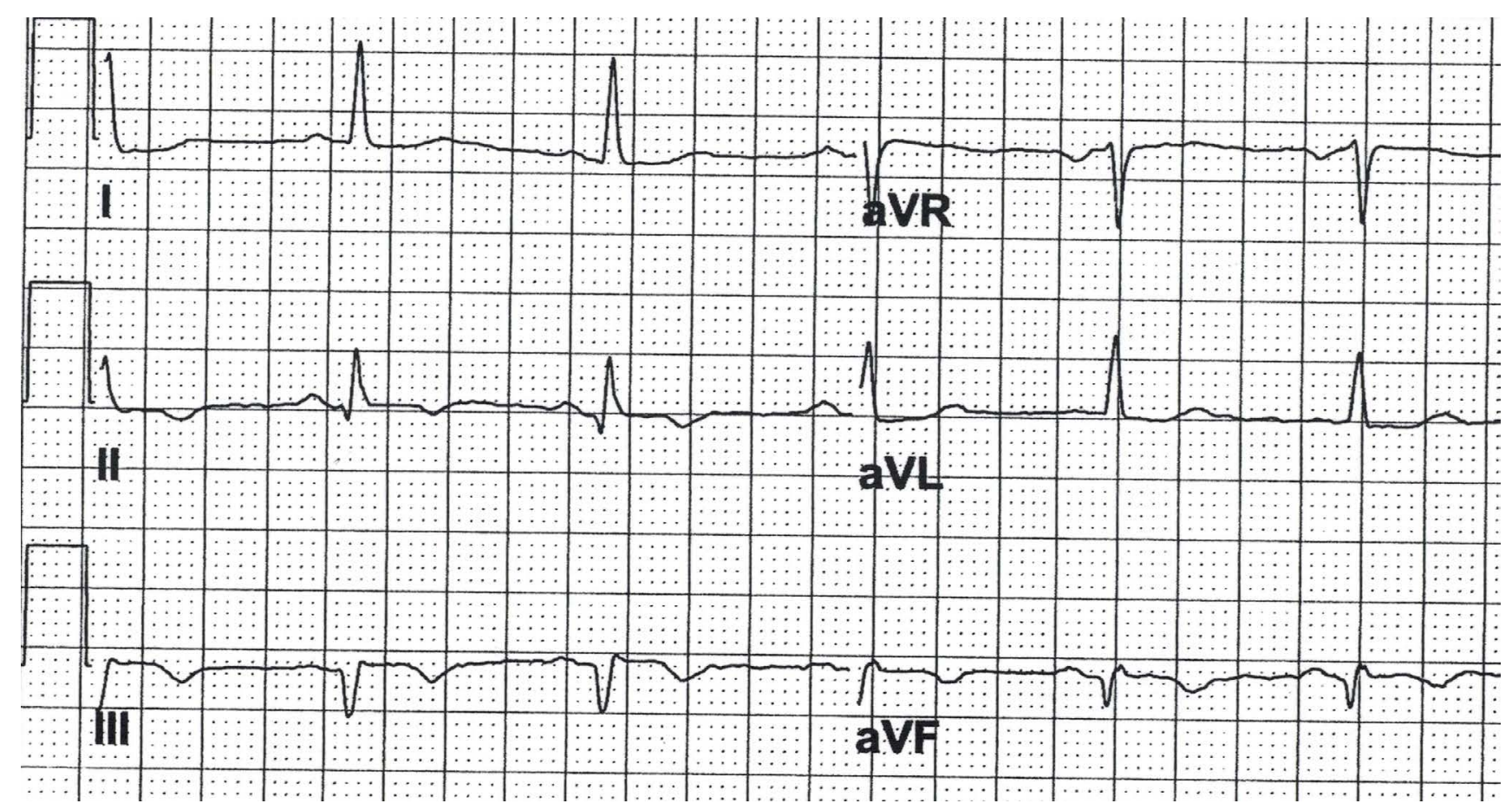

Figure 2: Abnormal $\mathrm{Q}$ waves (Duration $>40 \mathrm{msec}$. Amplitude $-0.02 \mathrm{mV}$ ) with T-wave inversions. 
repolarization abnormalities but imaging studies were normal.

\section{Discussion}

On a routinely performed ECG, computerized interpretation may suggest a diagnosis of an old IMI on the basis of the Q waves in the lead II, III and aVF. This may lead to further evaluation by imaging studies, consultations and rescheduling of elective procedures.

Historically, there has been a poor correlation between the computer-generated ECG diagnosis of an old IMI and the confirmed diagnosis of an IMI by imaging studies [5]. In our study the PPV was $52.8 \%$. Similar observations have been made by others [6]. Availability of information regarding a patient's clinical history and risk factors for $\mathrm{Ml}$ could enhance the interpretation of the ECG, however these variables are often not available to the clinician reading the ECGs.

Cardiac MRI is a reliable noninvasive reference standard for the identification and quantification of myocardial infarction In the studies comparing the presence of $Q$ wave with percent scar tissue on contrast-enhanced MR images, there is generally a good correlation $[6,7]$. If the infarction involved more than 10 to $15 \%$ of the left ventricular myocardium, the likelihood increases that the $Q$ waves would be detected on the electrocardiogram. Routine performance of cardiac MRI in the general population for the diagnosis of an old $\mathrm{MI}$ is not feasible, while ECG is an inexpensive and readily available tool.

Studies utilizing cardiac MRI for the diagnosis of MI and its comparison with ECG diagnosis of IMI were not segment specific. In the study performed by Asch, et al. only 23 out of 146 patients with IMI were identified [5]. In most of these studies cardiac MRI was performed within 2 to 3 weeks of the confirmed diagnosis of acute MI. The study population and the methodological approach were quite different from our patient population and our approach. In our study the objective was to identify parameters that could enhance the accuracy of the electively performed ECG for the diagnosis of old IMI.

Pathologic Q waves are related to the size of the MI [8,9]. Large Mls which involve more than $10 \%$ of the ventricular myocardium often exhibit significant $Q$ waves, while small infarcts may occur without leaving residual pathologic $Q$ waves on electrocardiogram [10]. This may explain why in some patients with a history of previous MI the ECG findings may not correlate [10]. Stratification based on $\mathrm{Q}$ wave area has been shown to predict the size of MI [11]. However, methods for calculating the area of the $Q$ wave are rather cumbersome and therefore are limited in their usefulness.

Q waves in electrocardiograms have been noted in conditions other than $\mathrm{Ml}$ such as healthy athletes, hy- pertrophic cardiomyopathy [12], pulmonary disease, preexcitation utilizing posteroseptal accessory pathway, and in the second and third trimester of pregnancy. Transient $Q$ waves may be present in intermittent preexcitation [13], misplaced arm lead electrodes or ischemia [14].

In left ventricular hypertrophy and hypertrophic cardiomyopathy $\mathrm{Q}$ waves are generally present in lateral leads. $Q$ waves of $\geq 40$ msec duration or $>3 \mathrm{~mm}$ depth in the inferior and lateral leads have been considered to be an abnormal finding requiring evaluation to rule out hypertrophic cardiomyopathy prior to participation in competitive sports. $Q$ waves, particularly in limb lead $\mathrm{I}$, can be used to distinguish $\mathrm{Q}$ waves in healthy athletes from patients with hypertrophic cardiomyopathy.

It has been speculated that the presence of $Q$ waves on the surface ECG carries an adverse prognosis $[15,16]$. Pathologic $Q$ waves due to myocardial scar may carry adverse prognostic implications, however physiologic $Q$ waves appear to be benign $[17,18]$. The PPV of the $Q$ wave alone in inferior leads of the ECG for the diagnosis of old MI appears to be low. In the presence of a scar from previous myocardial infarction, the $Q$ waves may reflect the electrical neutrality of the scar tissue. However, in other situations the $Q$ waves may be a reflection of the position of the heart in the chest and orientation of the electrical vector for a given lead.

If the $Q$ waves in a given ECG leads, are due to orientation and direction of the electrical vector, one can speculate that there may not be repolarization abnormalities in those leads. Such $Q$ waves may be more representative of normal anatomic variation than actual pathology while pathologic $Q$ waves may be accompanied by repolarization abnormalities [19]. Our results are consistent with this hypothesis. Our finding indicates that the patients with abnormal $Q$ waves and repolarization abnormalities in the same ECG leads tend to have abnormal imaging studies (PPV of $83.3 \%$ ).

Persistent $\mathrm{T}$ wave inversion, after the resolution of the ST elevation in patients with acute $\mathrm{MI}$, is considered pathologic. These changes may have diagnostic and prognostic implications [8].

If ECG showed significant $Q$ waves and T-wave inversions in inferior leads, as shown in Figure 2, then further evaluation would be appropriate. In the absence of significant $Q$ waves and repolarization abnormalities that interpretation could be revised. This could result in saving of valuable resources and alleviating the psychological distress of the patient.

Limitations: This was a retrospective collection and analysis of the data. Although large numbers of electrocardiograms were reviewed, the number of patients who had at least one imaging study to be included in this study was a small. 


\section{Conclusions}

Computer interpretation of ECGs can often result in a high rate of false positive readings for old IMI. The presence of abnormal $Q$ waves, if accompanied by repolarization abnormalities in the same leads, enhances the diagnostic accuracy of the electrocardiogram. Judicious use of imaging studies in this group of patients, if necessary, could eliminate, in a substantial number of patients, the false positive diagnosis of IMI and may help reduce the economic costs and psychological burden.

\section{Authors Declaration}

All the authors declare that there was no conflict of interest.

\section{Funding}

There was no funding involved in the research and preparation of this project.

IRB institution: Paul foster school of medicine, Texas tech University health sciences Center. El Paso Texas

IRB \#: E15047 SUBMISSION REFERENCE \#: 059266

\section{References}

1. Thygesen K, Alpert JS, Jaffe AS, Chaitman BR, Bax JJ, et al. (2018) Fourth Universal Definition of Myocardial Infarction (2018). J Am Coll Cardiol 72: 2231-2264.

2. Anderson JL, Morrow DA (2017) Acute Myocardial Infarction. N Engl J Med 376: 2053-2064.

3. Abedin Z, Conner RP (2007) ECG Interpretation: The Self-Assessment Approach. John Wiley and Sons.

4. Marriott HJL, Wagner GS (1988) Practical electrocardiography. Williams \& Wilkins.

5. Asch FM, Shah S, Rattin C, Swaminathan S, Fuisz A, et al. (2006) Lack of sensitivity of the electrocardiogram for detection of old myocardial infarction: A cardiac magnetic resonance imaging study. Am Heart J 152: 742-748.

6. Kaandorp TAM, Bax JJ, Lamb HJ, Viergever EP, Boersma $E$, et al. (2005) Which parameters on magnetic resonance imaging determine $Q$ waves on the electrocardiogram? Am J Cardiol 95: 925-929.

7. Florian A, Slavich M, Masci PG, Janssens S, Bogaert J (2012) Electrocardiographic Q-wave 'remodeling' in reperfused ST-segment elevation myocardial infarction: Validation study with CMR. JACC Cardiovasc Imaging 5: 10031013.
8. Wilkins ML, Maynard C, Annex BH, Clemmensen P, Elias WJ, et al. (1997) Admission prediction of expected final myocardial infarct size using weighted ST-segment, $Q$ wave, and T wave measurements. J Electrocardiol 30: 1-7.

9. Herlitz J, Hjalmarson A (1985) The relationship between the electrocardiographically estimated infarct size and 1and 2-year survival in acute myocardial infarction. Clin Cardiol 8: 141-147.

10. Ramos R, Albert X, Sala J, Garcia-Gil M, Elousa R, et al. (2016) Prevalence and incidence of $Q$-wave unrecognized myocardial infarction in general population: Diagnostic value of the electrocardiogram. The REGICOR study. Int J Cardiol 225: 300-305.

11. Kochav JD, Okin PM, Wilson S, Afroz A, Renilla A, et al. (2013) Usefulness of Q-wave area for threshold-based stratification of global left ventricular myocardial infarct size. Am J Cardiol 112: 174-180.

12. Bent RE, Wheeler MT, Hadley D, Froelicher V, Ashley E, et al. (2015) Computerized $Q$ wave dimensions in athletes and hypertrophic cardiomyopathy patients. J Electrocardiol 48: 362-367.

13. Letts DP, Constantine JC, Littmann L (2003) Intermittent noninfarction $Q$ waves: $A$ finding suggestive of latent preexcitation. Mayo Clin Proc 78: 840-843.

14. Barold SS, Falkoff MD, Ong LS, Heinle RA (1987) Significance of transient electrocardiographic $Q$ waves in coronary artery disease. Cardiol Clin 5: 367-380.

15. Birnbaum Y, Chetrit A, Sclarovsky S, Zlotikamien B, Herz I, et al. (1997) Abnormal $Q$ waves on the admission electrocardiogram of patients with first acute myocardial infarction: Prognostic implications. Clin Cardiol 20: 477-481.

16. Perino AC, Soofi M, Singh N, Aggarwal S, Froelicher $V$ (2015) The long-term prognostic value of the $Q$ wave criteria for prior myocardial infarction recommended in the universal definition of myocardial infarction. J Electrocardiol 48: 798-802.

17. Bogossian H, Ninios I, Frommeyer G, Mijic D, Hasan F, et al. (2015) Q Wave in the inferior leads: There is more than scar. Ann Noninvasive Electrocardiol 20: 609-611.

18. Moon JCC, Arenaza DPD, Elkington AG, Taneja AK, John AS, et al. (2004) The pathologic basis of Q-wave and nonQ-wave myocardial infarction: A cardiovascular magnetic resonance study. J Am Coll Cardiol 44: 554-560.

19. Reindl M, Reinstadler SJ, Feistritzer HJ, Niess L, Koch C, et al. (2017) Persistent T-wave inversion predicts myocardial damage after ST-elevation myocardial infarction. Int $\mathrm{J}$ Cardiol 241: 76-82. 